\title{
Semiconductor Nanorod Liquid Crystals and their Assembly on a Substrate
}

\author{
Liang-shi Li and A. Paul Alivisatos \\ Department of Chemistry, University of California, Berkeley \\ Materials Sciences Division, Lawrence Berkeley National Lab \\ Berkeley, CA 94720
}

Tremendous effort is presently devoted to the exploration of novel electrical and optical properties of one-dimensional nanoscale materials such as nanowires, nanotubes and nanorods $s^{12}$. The spatial organization of one-dimensional nanostructures requires a different set of methods from those that have been applied to spherical or small aspect ratio nanocrystals ${ }^{45}$. In some instances, it is desirable to place individual nanowires in a particular configuration ${ }^{6}$. In the present case, our interest is focused on large scale spatial organization of nanorods. Some methods that have been applied to such assembly of nanorods and nanowires include the Langmuir-Blodgett technique ${ }^{78}$ and simultaneous synthesis with assembly ${ }^{9}$, but alignment and patterning on a macroscopic scale are still very difficult to achieve. On the other hand, it has been well recognized since Onsager's pioneering work that suspension of rod-like particles may form lyotropic crystalline phases $^{10}$, in which the rod-like particles on average have a common orientation but no or only partial positional order. Lyotropic liquid crystals made of suspensions of organic macromolecules or rod-like viruses ${ }^{11,12}$ have been known for decades and extensively investigated. A few examples of lyotropic liquid crystalline phases completely consisting of inorganic minerals have also been studied ${ }^{13}$, but mainly for fundamental understanding of liquid crystal formation. Recent synthetic advances in the preparation of inorganic nanoparticles with anisotropic shapes have yielded a wide variety of systems with interesting electronic, optical and magnetic properties ${ }^{14} 151617$, and alignment of them is desired for both fundamental study and applications. In this paper we describe recent studies of alignment and surface deposition from the liquid crystalline phase of $\mathrm{CdSe}$ nanorods.

We chose to study CdSe nanocrystals because their growth is very well controlled ${ }^{18}$, and their electro-optical properties are well understood ${ }^{19}$. The latest development in synthesis has made it possible to synthesize rod-like CdSe nanocrystals with tightly 
controlled width and length. ${ }^{20}$ It has also been demonstrated that these CdSe nanorods have photoluminescence linearly polarized along the long axis ${ }^{3}{ }^{14}$. In order to align the CdSe nanorods on a large scale $(\mathrm{mm})$, we take advantage of the tendency of rod-like objects to form lyotropic phases. When the nanorods are dissolved in cyclohexane to make a very concentrated solution, spontaneous formation of a nematic phase is observed, as identified from the Schlieren structures under an optical polarizing microscope $\mathrm{e}^{21}$. In the liquid crystalline phase the nanorods exhibit cooperative phenomena that extend the available methods for aligning the nanorods, for instance, using external magnetic or electric fields, or by interaction with a surface. To demonstrate the liquid crystalline character of these solutions, we use a combination of wide and small angle $\mathrm{X}$ ray diffraction. Figure 1 shows the wide-angle x-ray diffraction (1(A)) and small angle X-ray scattering patterns (1(B)) from a nematic solution in a 300-micron thick capillary tube. These rods have a length of $\sim 60 \mathrm{~nm}$ and width of $3.0 \mathrm{~nm}$. In both figures the capillary tube remains vertical. In figure 1 (A) the sharp arcs in the vertical position are due to (002) planes of the CdSe crystalline lattice within individual nanorods. The high intensity and sharpness of the arcs are determined by the elongated shape of the CdSe nanorods ${ }^{14}$. The anisotropy of the pattern demonstrates macroscopic alignment of the long axis of the rods. Consistently, in figure 1(B) the diffuse arcs in the equator direction correspond to the lateral distance of the nanorods, suggesting the short axis is aligned as well. Together, the patterns demonstrate the preferential alignment of the CdSe nanorods along the capillary tube axis direction, which is attributed to the curved surface of the capillary tube. It can be estimated that about $70 \%$ of the nanorods are oriented within $20^{\circ}$ from the axis of the capillary tube. More work on alignment of bulk phases with external fields is in progress.

For various applications such as polarized light emitting diodes ${ }^{22,23,24}$, large areas of alignment of nanorods on a substrate are required. Thus it is of considerable interest to learn more about how nanocrystal superlattices nucleate on a substrate and how the nucleation events may potentially be controlled. Here we use transmission electron microscopy (TEM) to study the structures formed after CdSe nanorods are deposited on a substrate from a solution by slow solvent evaporation. 
In order to obtain structures thin enough for TEM study, a freshly cleaved mica sheet coated with a thin carbon film is immersed vertically into a dilute solution of CdSe nanorods in cyclohexane. As the solvent evaporates, the liquid level sweeps over the substrate, depositing CdSe nanorods behind when the solvent is completely gone. The convective flow caused by the solvent evaporation carries the nanorods from the bulk solution to the liquid-solid-air interface and can make the solution locally very concentrated $^{25}$, and therefore phenomena such as nanorod crystallization can occur. The carbon film is then floated off on water and picked up on a TEM grid. We also used commercial carbon coated copper grids but the results are the same.

Because of the inhomogeneity in local concentration and the nonequilibrium nature of the solvent evaporation method, assemblies with different levels of order can be left on the carbon film, ranging from amorphous to crystalline. Figure 2 shows the typical structures observed in ordered regions for $3.7 \mathrm{~nm} \times 18 \mathrm{~nm}$ CdSe nanorods in different areas on the same TEM grid. Figure 2(A) shows an area formed by a nematic liquid crystalline nanorod phase simply collapsing onto the substrate without much rearrangement, in which the nanorods are positioned randomly but aligned overall in the same direction. 2(B) and 2(C) show two different types of crystalline structures. In 2(B) a multi-layered structure is shown, with CdSe nanorods connecting to each other head-totail to form linear arrays. Different layers appear to be different domains. Due to the overlap between different layers and the finite length distribution of these nanorods, no overall periodicity is observed along the direction of the line structure. Within each domain periodicity along the direction perpendicular to the line directions can be seen, with an interline spacing of $\sim 1.1 \mathrm{~nm}$, which corresponds to twice the length of the capping molecules on the nanocrystals. In each line the nanorods are closely packed. This structure is particularly interesting because in each domain the lines have a high degree of positional order, and by replacing the surfactants on the surface of these nanorods with other molecules, we may control the distance between these lines.

In the linear array structures very frequently vortex structures are observed, as shown in figure $3(\mathrm{~A}-\mathrm{C})$. In particular, figure $3(\mathrm{C})$ demonstrates an isolated vortex structure, together with its small angle electron diffraction pattern, suggesting its spontaneous formation on the amorphous substrate. The change of orientations of nanorods around the 
vortex clearly resembles a disclination of strength +1 in a nematic liquid crystalline phase $^{26}$, so we believe these vortex structures formed from the nematic phase. Recently similar disclination structures have also been observed in $\mathrm{BaCrO}_{4}$ nanorod assemblies with $\mathrm{TEM}^{8}$, in which the high compression in the Langmuir film preserves the distortion from further relaxation. In our case the CdSe nanorods around the cores of these vortex structures are divided into six branches, which can be attributed to the difference between Frank (elastic) ${ }^{27}$ constants for different types of distortions. According to Onsager's rigid rod theory, when the solution is very concentrated, the Frank constants for splay and for bend become much larger than that for twist ${ }^{28,29}$. Therefore the rods in the vicinity tend to break into different layers to lower the total elastic energy during the drying. Across the boundaries of two neighboring branches, a discontinuity of $\sim 60^{\circ}$ in orientation appears. Furthermore, each branch around a defect is a distinct crystalline domain, so we conclude that the cores of the vortices are the nucleation sites for the linear arrays. It has been well known in natural crystals that defects play important roles in crystal nucleation and growth. The existence of defects significantly lowers the energy barrier for heterogeneous nucleation. Similarly, the vortex patterns observed here appear to nucleate from defects in the nanorod liquid crystalline phase, and extend into the linear arrays shown in figure 2 (B). Formation of linear arrays from a nematic phase was also observed for larger rodlike particles, e.g. tobacco mosaic virus, with a combination of polarizing optical microscopy and atomic force microscopy $(\mathrm{AFM})^{30}$. Interestingly, in the linear arrays of tobacco mosaic viruses, a critical bending angle of $60^{\circ}$ was also observed, which is accompanied by breaking of individual virus particles and attributed to the elastic limit. In our case, the strain caused by the discontinuity of $60^{\circ}$ between the domains is relaxed by the twisting between different layers (domains). The reason for the presence of the $60^{\circ}$ critical angle in our system, however, is not yet understood.

The monolayer structure with periodicity in both directions, as shown in 2(C) may have originated from a smectic-A phase. Numerical simulation on rigid rods has revealed the possibility of formation of smectic phases, and this has been confirmed experimentally in a few systems ${ }^{13}$. Similar structures have been observed with AFM study of the smectic layers formed from dry sol sediments of needle-like $\beta$-FeOOH particles $^{31}$, which are well known for forming a smectic phase in aqueous suspension. 
Even though so far we do not have any direct evidence of smectic phases in $\mathrm{CdSe}$ nanorod solutions, the superlattice structures observed here suggest their possible presence. It is believed that $\mathrm{CdSe}$ nanorods have a permanent dipole moment along the long axis, and numerical study has shown a longitudinal dipole may stabilize smectic phases with respect to the nematic phase ${ }^{32}$.

In summary, we have shown the macroscopic alignment of CdSe nanorods in a nematic liquid crystalline phase, and that to a great extent the superlattice structures of these nanorods formed upon deposition on a substrate are determined by the liquid crystalline phases that formed prior to complete solvent evaporation. Because of the high controllability of liquid crystalline phases by external fields or pretreated surfaces, by controlling the phase of liquid crystalline solution and its orientation we may ultimately be able to achieve a high degree of control over the deposited films of the nanorods.

Acknowledgement We thank Mario Yosa and Youli Li at the Material Research Laboratory (MRL) of University of California at Santa Barbara for the assistance in the X-ray scattering measurement. This work was supported in part by the Director, Office of Science, Office of Basic Energy Sciences, Division of Materials Sciences and Engineering of the U.S. Department of Energy under Contract No. DE-AC03-76SF00098 and in part by the Air Force Office of Scientific Research under Grant No. F49620-01-10033. 


\section{Figure caption}

Figure1. Wide angle x-ray diffraction and small angle $x$-ray scattering of the same nematic solution of CdSe nanorods in a $300 \mu \mathrm{m}$ thick capillary tube show the preferential alignment of these nanorods along the axis of the capillary tube. In both measurements the capillary tube is held vertically. A) Wide angle x-ray diffraction pattern. The vertical sharp, intense arcs correspond to the (002) planes of wurzite CdSe lattice. At about the same radius weak arcs corresponding to the (100) and (101) planes can also be seen, but they are much more diffuse. B) Small angle x-ray scattering pattern. The two diffuse arcs at $\mathrm{Q} \sim 1.5 \mathrm{~nm}^{-1}$ on the equator corresponds to the lateral spacing between the rods in the nematic phase. The arcs corresponding to the longitudinal spacing are not resolved.

Figure 2. Transmission electron micrograph images of typical superlattice structures formed when CdSe nanorods are deposited on a carbon film from a solution by slow solvent evaporation method. All the nanorods are $3.7 \mathrm{~nm}$ in diameter and $18 \mathrm{~nm}$ in length. A) Structures in which the nanorods have an overall orientational order but random positions. B) Multi-domain linear arrays. Within each domain, the nanorods have lateral positional order but no longitudinal order. Different domains have a $\sim 60^{\circ}$ angle in orientation. C) Monolayer in which the rods have positional order in both lateral and longitudinal directions.

Figure 3. Vortex structures in the linear arrays. In all of the vortex structures, six branches can be identified. It is believed that the linear arrays nucleate at these vortices, and each branch of them evolves into a domain. Figure 3C) shows an isolated vortex, suggesting the spontaneous formation of these structures. The inset is the small angle electron diffraction pattern of the selected area. It clearly shows the six-fold symmetry of the packing. 
${ }^{1}$ J Hu, T. W. Odom, C. M. Lieber, Acc. Chem. Res. 1999,32, 435.

2 J. Wang, et al., Science 2001, 293, 1455.

${ }^{3}$ J. Hu, et al., Science 2001,292, 2060.

${ }^{4}$ C. B. Murray, C. R. Kagan, M.G. Bawendi, Science 1995,270, 1335.

${ }^{5}$ C. A. Mirkin, et al., Nature 1996,382, 607.

${ }^{6}$ C.M. Lieber, Sci. Am. 2001, 285, 58.

${ }^{7}$ B. O. Dabbousi, C. B. Murray, M. F. Rubner, M. G. Bawendi, Chem. Mat. 1994, 6, 216.

${ }^{8}$ F. Kim, S. Kwan, J. Akana, P. D. Yang, J. Am. Chem. Soc. 2001,123, 4360.

${ }^{9}$ M. Li, H. Schnablegger, S. Mann, Nature 1999,402, 393.

${ }^{10}$ I. Onsager, Ann. N. Y. Acad. Sci. 1949, 51, 627.

${ }^{11}$ C. Robinson, J. C. Ward, Nature 1957,180, 1183.

${ }^{12}$ F. C. Bowdwen, et al., Nature 1936,138, 1051.

${ }^{13}$ A. S. Sonin, J. Mater. Chem. $1998,8,2557$.

${ }^{14}$ X. Peng, et al., Nature 2000, 404, 59.

${ }^{15}$ T. S. Ahmadi, et al., Science 272, 1924 (1996).

${ }^{16}$ V. F. Puntes, K. M. Krishnan, A. P. Alivisatos, Science 2001, 291, 2115.

${ }^{17}$ S. Chang, et al., Langmuir 1955, 15, 701.

${ }^{18}$ C. B. Murray, D. J. Norris, M. G. Bawendi, J. Am. Chem. Soc. 1993,115, 8706.

${ }^{19}$ Al. A. Efros, M. Rosen, Annu. Rev. Mater. Sci. 2000, 30, 475.

${ }^{20}$ L.-S. Li, J, Hu, W. Yang, A. P. Alivisatos, Nano Lett. 2001, 1, 349.

${ }^{21}$ L.-S. Li, J. Walda, L. Mann, A. P. Alivisatos, Nano Letters. 2002, 2, 557.

${ }^{22}$ V. L. Colvin, M. C. Schlamp, A. P. Alivisatos, Nature 1994, 370, 354.

${ }^{23}$ M. Era, T. Tsutsui, S. Saito, Appl. Phys. Lett. 1995, 67, 2436.

${ }^{24}$ P. Dyreklev, et al., Adv. Mat. 1995, 7, 43.

${ }^{25}$ N. D. Denkov, et al Nature 1993, 361, 26.

${ }^{26}$ S. Chandhrasaka, Liquid Crystals, Cambridge University Press, 1992.

${ }^{27}$ F. C. Frank, Disc. Faraday Soc. 1958, 25, 19.

${ }^{28}$ R. G. Priest, Phys. Rev. A 1973, 7, 720.

${ }^{29}$ Straley, J. P. Phys. Rev. A 1973, 8, 2181.

${ }^{30}$ H. Maeda, Langmuir 1997, 13, 4150.

${ }^{31}$ H. Maeda and Y. Maeda, Langmuir 1996, 12, 1446.

${ }^{32}$ S. C. McGrother, A. Gil-Villegas, G. Jackson, Mol. Phys. 1998, 95, 657. 
Fig. 1
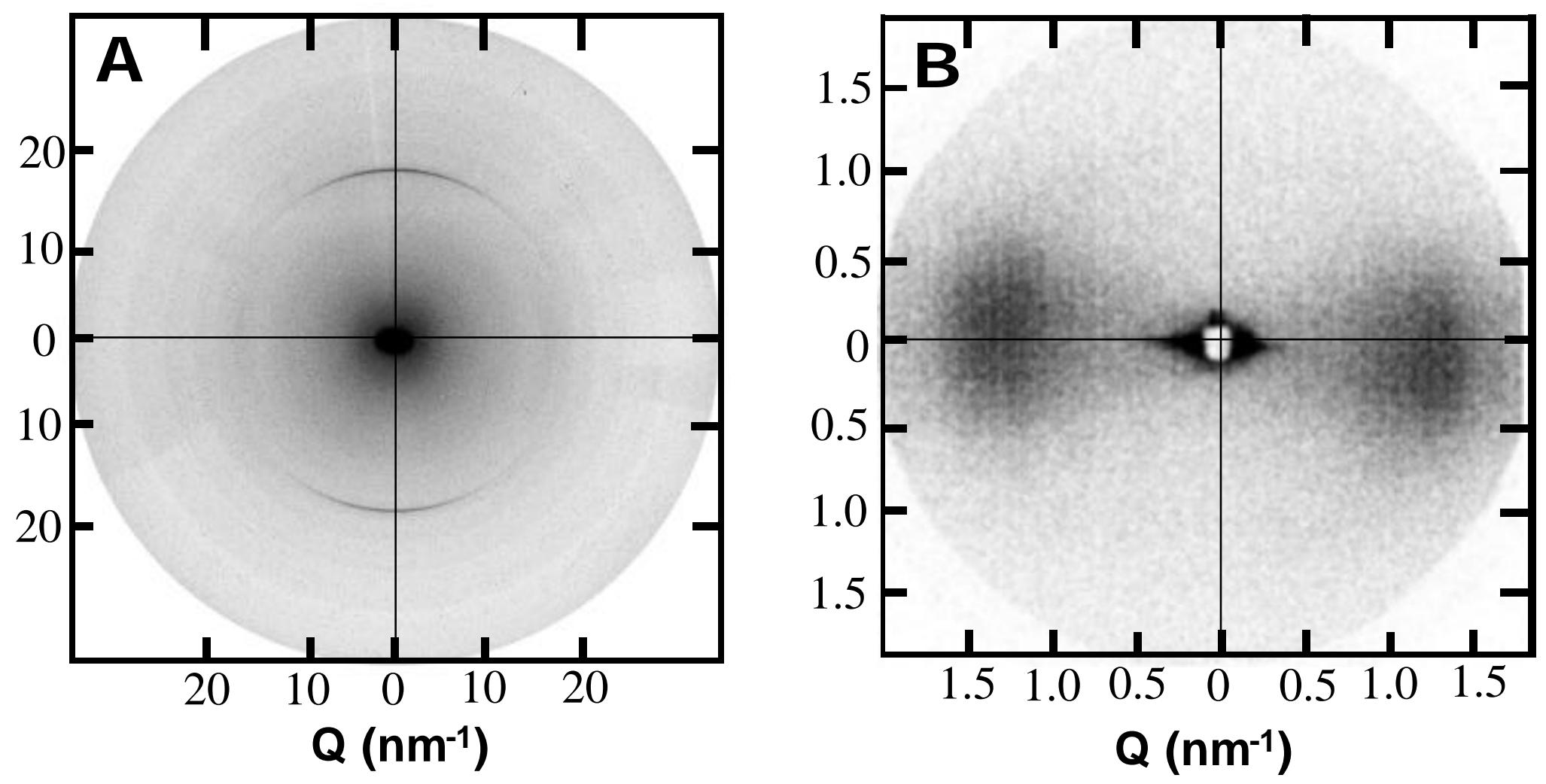
Figure 2
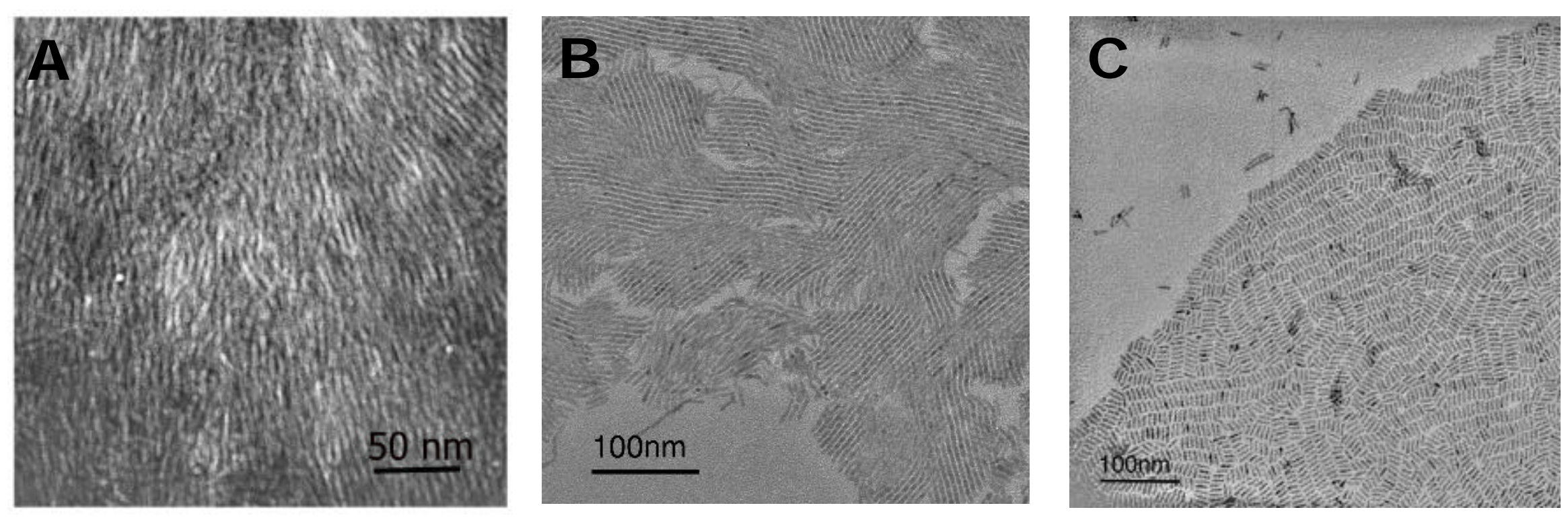
Figure 3
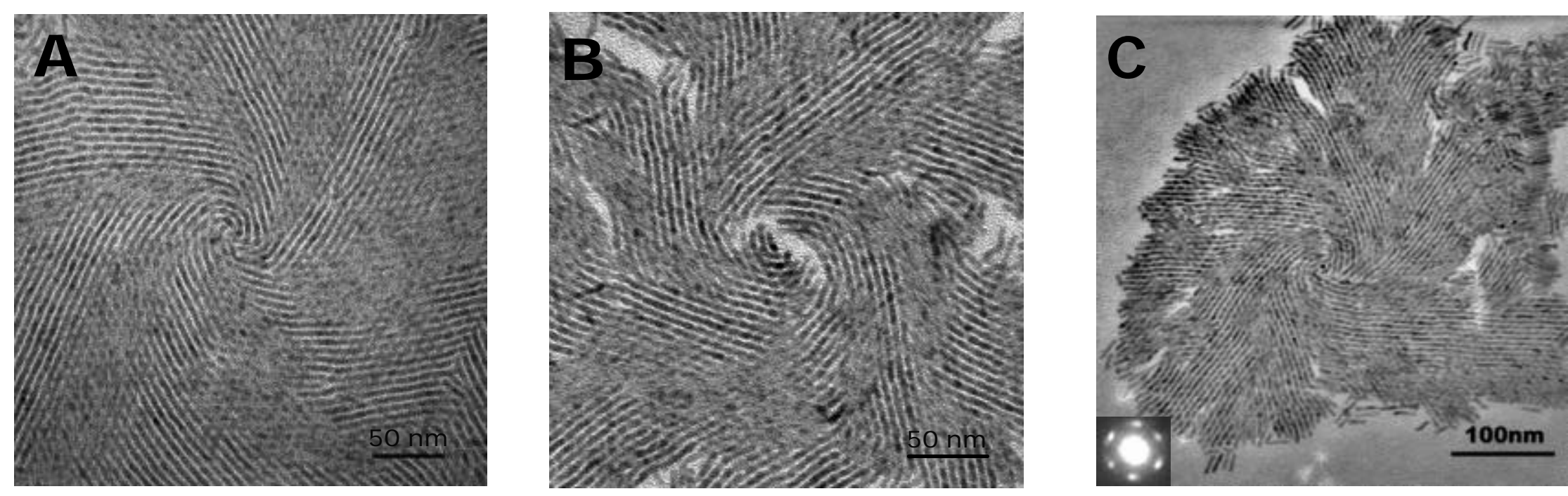